Finalmente cabe destacar que el tiempo transcurrido nos permite apreciar la lucidez de Pietilä para ver que, en el futuro, la problemática de la arquitectura quedaría necesariamente unida al problema de la sostenibilidad urbana y que éste habría que abordarlo desde una nueva perspectiva ecológica capaz de integrar lo social, lo cultural y lo ambiental. Entendiendo que la capacidad de transformación de esta nueva visión del mundo dependía y requería de una nueva forma de abordar la docencia en las escuelas de arquitectura. En un momento como el actual, en el que se debate sobre la importancia de la formación del arquitecto en relación a las expectativas y valores de la sociedad del siglo $X X I$, resulta relevante recordar experiencias como las de Reima Pietilä, surgidas del feliz encuentro entre una labor docente reflexiva y una práctica profesional abierta a la investigación, como un camino de referencia para abordar los retos del futuro. "Concibe una contracultura" era la provocadora recomendación final que Reima Pietilä hizo a sus alumnos de Oulu en Notion Image Idea.

1 Estos textos fueron escritos entre 1973 y 1974, entregados en 1974 y publicados a modo de cuaderno pedagógico en 1975: PIETLA, Reima. Notion Image Idea. Espoo: Teknillisen organizó junto con el grupo Dimensio una exposición basada en estas reflexiones, titulada también Notion Image Idea.

2 Esta definición de Pietilä de Notion Image Idea se recoge en: QUANTRILL, Malcolm. Reima Pietilä. Architecture, context and

${ }^{3}$ PALLASMAA, Juhani., "Reima Pietilä y el círculo del museo de arquitectura finlandesa". En: Raili : Reima Pietila. Un desafio a
arquitectura moderna. Madrid: Fundación ICO, 2008, p.20

${ }^{4}$ Sobre el constructivismo finlandés ver: AA.W. Forma y estructura. El constructivismo en el arte moderno, en la arquitectura y en las artes aplicadas finlandesas. Madrid: 1980.

5 AALTO, Alvar. "La trucha y el torrente de montaña". En: SHILDT, Gorän. Alvar Aalto. De palabra y por escrito. El Escorial: El

${ }^{6}$ BLOMSTEDT, Aulis. “Canon 60”. Le Carré Bleu. 1961, núm.4, p. 25 .

7 Reima Pietilä publicaba regularmente en la revista Le Carré Bleu, de la que era cofundador con Aulis Blomstedt, y en Arkkiteh puede consultarse en: QUANTRII 1 Malcolm Op cit. 246.

${ }^{8}$ Frase inicial de la Introducción de Notion Image Idea en la versión en finés. Se recoge en: QUANTRILL, Malcolm. Op.cit.

${ }^{9}$ Las referencias al juego como forma de investigación son frecuentes en sus escritos ver: QUANTRILL, Malcolm. Op.cit. Jardín, presentada en el Museo de Arquitectura Finlandesa de Helsinki en 1971.

${ }^{10}$ PIETILÄ, Reima. Op.cit. p.1.

${ }^{11}$ lbíd. p.2.

2 Reima Pietilä en conversación con Kaisa Broner-Bauer. Ver: 1995, núm. 2, p.7.

${ }^{13}$ PIETILÄ, Reima. Op.cit. p.3.

${ }^{14}$ ENGELS, Friedrich. Dialéctica de la naturaleza. Madrid: Akal, 2017

${ }^{15}$ HUIZINGA, Johan. Homo ludens. Madrid: Alianza, 2007.

BIBLIOGRAFÍA CITADA

AALTO, Alvar. "La trucha y el torrente de montaña". En: SHILDT, Gorän. Alvar Aalto. De palabra y por escrito. El Escorial: El Croquis, 2000.

AA.W. Forma y estructura. El constructivismo en el arte moderno, en la arquitectura y en las artes aplicadas finlandesas. Museos, 1980.

AA.W. "Price, Culot, Pietilä. De la pasión por la tierra". Fisuras. 1995, núm. 2 .

BLOMSTEDT, Aulis. "Canon 60”. Le Carré Bleu. 1961, núm. 4.

ENGELS, Friedrich. Dialéctica de la naturaleza. Madrid: Akal, 2017.

HUIZINGA, Johan. Homo ludens. Madrid: Alianza, 2007 PALLASMAA, Juhani. "Reima Pietilä y el círculo del museo de arquitectura finlandesa". En: Raili : Reima Pietilä. Un desafío a la arquitectura moderna. Madrid: Fundación ICO, 2008 Korkeakoulun Ylioppilaskunta, 1975.

QUANTRILL, Malcolm. Reima Pietilä. Architecture, context and modernism. Helsinki: Otava, 1984.

ENRIQUE FERNÁNDEZ-VIVANCOS GONZÁLEZ es doctor arquitecto y profesor asociado del Departamento de Proyectos Arquitectónicos de la ETSA, Universitat Politècnica de València (UPV).

\title{
Dobles espacios superpuestos
}

\author{
Miguel Centellas, Pere Joan Ravetllat
}

Recibido 2019.06.11 :.: Aceptado 2019.06.12

DOI: 10.5821/palimpsesto.19.8526

ABSTRACT superpuestos, el número 04 de la colección "Temas de Vivienda" impulsada por el grupo de investigación "Habitar Colectivo" de la Universidad Politécnica de Cartagena y dedicada a glosar los diferentes parámetros que definen la configuración de la vivienda. La monografia propone revisitar un conjunto de edificios residenciales que comparten la condición especial de albergar un domésticas. Edificios no convencionales que han buscad transformar la vivienda y su relación con la ciudad a través de un doble espacio superpuesto.

PALABRAS CLAVE: vivienda; proyecto; doble espacio.

Persona de contacto: centellasmiguel@gmail.com. Departamento de Arquitectura y Tecnología de la Edificación de la Escuela Técnica Superior de Arquitectura y Edificación (UPCT).

a colección "Temas de Vivienda" impulsada por el Grupo de Investigación "Habitar Colectivo" de la Universidad Politécnica de Cartagena, recoge, en diferentes volúmenes, un estudio detallado de los distintos parámetros que definen la configuración de la vivienda a través de ejemplos de proyectos en el marco de la arquitectura española, aunque en el último número se estudian ejemplos internacionales.

La investigación analítica y crítica de forma fragmentada sobre las herramientas arquitectónicas que construyen el espacio en el que se desarrollan los diferentes modos de habitar, resulta de interés dentro del panorama actual de la arquitectura y, en especial, en su estrecha relación con los actuales cambios sociales, económicos, culturales, tecnológicos y energéticos.

El contenido de la colección se estructura en libros monográficos sobre la investigación en un tema concreto (fondo edificado, tipos, espacios intermedios, doble circulación, vivienda pasante, flexibilidad, simultaneidad de usos, diagonalización del espacio, variación de alturas libres, sistemas de compartimentación, envolventes, equipamiento como sistema de objetos, etc.) en el que se desarrolla de forma introductoria una síntesis a modo de conclusiones sobre el parámetro estudiado junto con un catálogo redibujado a la misma escala de obras y proyectos que ejemplifican, de la forma más amplia y posible, la utilización y puesta en práctica real de dicho elemento.

Desde 2014 se han editado los siguientes libros: 1. Fondo edificable; 2. Cuatro viviendas por rellano; 3. La vivienda adosada y 4. Dobles espacios superpuestos publicado en octubre de 2018. En esta nueva monografía, se propone revisitar un conjunto de edificios residenciales sobre los que reflexionar. La relación entre ellos no es "tipológica" ni una específica distribución como sucede en las tradicionales clasificaciones de los proyectos de vivienda. Tampoco responde a una época histórica, ni a una adscripción estilística a unos determinados parámetros. Los casos presentados comparten la condición especial de albergar un espacio que supera en altura las habituales dimensiones domésticas. Una característica fácil de encontrar en proyectos de baja densidad, pero menos usual cuando se reitera la planta tipo generando los Dobles Espacios Superpuestos.

A través del conjunto de edificios que se analizan puede apreciarse como los arquitectos han buscado acentuar el espacio en el interior proponiendo diversas soluciones. Edificios no convencionales que han buscado transformar la vivienda y su relación con la ciudad a través de un Doble Espacio Superpuesto.
Debido a la mayor complejidad volumétrica de dichos conjuntos que transforma su percepción espacial interna, se complementan las habituales plantas tipo mediante secciones y axonometrías. De este modo, proporciones, recorridos y visuales quedan reflejados en documentos tridimensionales explicativos de su configuración espacial.

Pueden analizarse distintas situaciones espaciales en relación al funcionamiento de la planta, a veces central, relacionado con el exterior o con la escalera y se ven las alteraciones de sección que inexcusablemente deben producirse. Pero quizás lo más sustancial del conjunto propuesto sea la condición de asumir al mismo tiempo una doble escala urbana y doméstica. Una simultánea participación del interior y del exterior que se aborda en la publicación.

Se han estudiado los siguientes edificios presentados por orden cronológico: Narkomfin en Moscú de Ginzburg y Milinis; viviendas en la calle Montaner de Sert; Highpoint II en Londres de Lubetkin ; estudios para artistas en Buenos Aires de Bonet Castellana; la Unidad de Habitación en Marsella de Le Corbusier; las viviendas en el barrio Hansa en Berlín de Pierre Vago; las de St. Jame's place en Londres de Lasdun; el edificio en la calle Reyes Magos en Madrid de Cabrero; Fregoli en Barcelona de Esteve Bonell; Nemausus en Nimes de Nouvel; las viviendas en el Paseo de Gracia de Barcelona de Ferrater y los Lofts Yerba Buena en San Francisco de Saitowitz-Natoma.

El conjunto de ejemplos analizados se ha construido en un periodo de unos setenta años (entre 1930 y 2002). Como se puede observar existe una gran diversidad en dimensiones, tipos y emplazamientos. Se muestran apartamentos muy pequeños (Bonet en Buenos Aires) y viviendas de gran superficie (Correa en Bombay). También es muy variado el tamaño de los edificios: desde los 8 estudios en el de Bonet a los más de 300 apartamentos de la Unidad de Habitación de Le Corbusier.

Algunos edificios se han desarrollado mediante bloques alargados y otros en altura. Se pueden observar ejemplos desde 4 plantas (Bonet) hasta 28 (Correa). Algunas viviendas están dispuestas entre medianeras, otras se sitúan en esquina, otras en el interior de un patio de manzana, como las de Ferrater. De los tipos seleccionados 6 presentan acceso por corredor y 7 mediante un núcleo vertical. A veces los dobles espacios son interiores a las viviendas aunque en la mayoría de las ocasiones vuelcan hacia el exterior.

Se ha realizado un recorrido por 13 ejemplos residenciales del siglo XX, en el que los arquitectos volcaron en los proyectos uno de los paradigmas arquitectónicos que ha diferenciado a los edificios del pasado siglo y que realmente sirve para potenciar una de las características más importantes de la arquitectura: la calidad del espacio interior. Sin duda, cada uno de los casos analizados son referentes destacados en el ámbito residencial.

MIGUEL CENTELLAS SOLER es Docto Arquitecto y Profesor Titular del Área de Proyectos Arquitectónicos del Departamento de Arquitectura y Tecnología de la Edificación de la ETSAE, Universidad Politécnica de Cartagena (UPCT). PERE JOAN RAVETLLAT es Doctor Arquitecto y Catedrático del Departamento de Proyectos Arquitectónicos de la ETSAB, Universitat Politècnica de Catalunya (UPC). 